Rev. Inst. Flor. v. 27 n. 2 p. $155-165$ dez. 2015

http://dx.doi.org/10.4322/rif.2015.012

ISSN impresso 0103-2674/on-line 2178-5031

\title{
SOBREVIVÊNCIA E CRESCIMENTO DE ESPÉCIES TROPICAIS MADEIREIRAS EM CONSÓRCIO COM ESPÉCIE PIONEIRA EM DIFERENTES PROPORÇÕES ${ }^{1}$

\author{
SURVIVAL AND GROWTH OF TROPICAL TIMBER SPECIES INTERCROPPED WITH \\ PIONEER SPECIES IN DIFFERENT PROPORTIONS
}

\author{
Caio Eduardo Melo de SOUZA², ${ }^{2}$ Osmar VILAS BÔAS ${ }^{3}$; Giselda DURIGAN³
}

\begin{abstract}
RESUMO - O cultivo de espécies florestais nativas com fins comerciais em plantios monoespecíficos vem sendo estudado há algumas décadas no Brasil. A silvicultura por meio de plantios consorciados, porém, raramente tem sido objeto de estudo. Avaliou-se o desempenho de duas espécies nativas de interesse comercial: a aroeira (Myracrodruon urundeuva) e o angico vermelho (Anadenanthera colubrina var. cebil), em consorciação com uma espécie pioneira, o capixingui (Croton floribundus), para verificar a influência de diferentes proporções da espécie pioneira na sobrevivência e crescimento das espécies de interesse comercial. Testaram-se as hipóteses de que diferentes proporções no plantio consorciado resultam em diferenças no crescimento das espécies e que a resposta ao plantio consorciado é diferente entre as espécies-alvo. O presente estudo foi realizado em dois experimentos, um para cada espécie-alvo, instalados em blocos ao acaso, com cinco repetições de cada tratamento $(25,50$, 75 e $85 \%$ da espécie pioneira), em Paraguaçu Paulista, SP. Quinze anos após o plantio, os sistemas consorciados apresentaram alguns padrões: 1) a mortalidade superior para a espécie pioneira em comparação com as espécies-alvo, 2) crescimento mais rápido das árvores das espécies-alvo quanto maior a proporção da espécie pioneira, 3) quanto maior a proporção de pioneiras, menor densidade final do sistema, resultando em menor competição e favorecendo o crescimento individual das espécies-alvo, e 4) não houve variação volumétrica por hectare das espécies-alvo que possa ser explicada pela proporção de pioneiras para nenhuma das duas espécies. Assim, embora a consorciação com espécies pioneiras favoreça o crescimento individual das espécies-alvo, não há nenhum efeito da consorciação no volume de madeira produzido por hectare.
\end{abstract}

Palavras-chave: silvicultura de espécies nativas; plantios mistos; competição; Anadenanthera colubrina var. cebil; Croton floribundus; Myracrodruon urundeuva.

\footnotetext{
${ }^{1}$ Recebido para análise em 12.08.15. Aceito para publicação em 01.12.15.

'Laboratório de Sistemática Vegetal, Departamento de Ciências Biológicas, UNESP, Faculdade de Ciências e Letras de Assis, Av. Dom Antônio, 2100, Parque Universitário, 19806-900 Assis, SP, Brasil.

${ }^{3}$ Instituto Florestal, Rua do Horto, 931, 02377-000 São Paulo, SP, Brasil.

${ }^{4}$ Autor para correspondência: Caio Eduardo Melo de Souza - caioeduardoms@hotmail.com
} 


\begin{abstract}
The growth of native forest species for commercial purposes in monospecific plantations has been studied for decades in Brazil. However, forestry through mixed stands has rarely been studied. We evaluated the performance of two native species of commercial interest: the aroeira (Myracrodruon urundeuva) and the angico vermelho (Anadenanthera colubrina var. cebil) in mixed stands with a pioneer species, the capixingui (Croton floribundus), to verify the influence of different proportions of the pioneer species in the survival and growth of the commercial species. The tested hypothesis was that intercropping in different ratios results in differences in their growth and that the response to the intercropping is different among the target species. The study was conducted in two experiments, one for each target species, installed in randomized blocks, with five repetitions of each treatment $(25,50,75$ and $85 \%$ of pioneer species) in Paraguaçu Paulista, state of São Paulo, Brazil. Fifteen years after planting, the intercropped systems showed some patterns and differences. Among the patterns: 1) pioneer species presented higher mortality in comparison to the target species, 2) the higher the proportion of pioneer species, the faster was tree growth of the target species, 3) the higher the proportion of pioneers, the lower the final density of the system, resulting in less competition and favoring growth of the target species and 4) the timber volume per hectare could not be explained by the proportion of pioneer species used in the system for none of the two focal species. So while intercropping with pioneer species favors the individual growth of the target species, there is no effect of intercropping on timber volume produced per hectare.
\end{abstract}

Keywords: tropical forestry; mixed stands; competition; Anadenanthera colubrina var. cebil; Croton floribundus; Myracrodruon urundeuva.

\section{INTRODUÇÃO}

O Estado de São Paulo possui oficialmente em seu território cerca de 24,8 milhões de hectares (Instituto Brasileiro de Geografia e Estatística - IBGE, 2014), dos quais 3,85 milhões $(15,5 \%)$ eram cobertos por formações florestais e outros tipos de vegetação nativa no início dos anos 1990. No levantamento mais recente (Sistema de Informações Florestais do Estado de São Paulo - SIFESP, 2010), tal valor aumentou cerca de $2 \%$, indo para 4,34 milhões de hectares de cobertura vegetal, sendo que deste total aproximadamente 1 milhão de hectares (23\%) consiste em áreas reflorestadas (São Paulo, 2008). Supondo que um valor mínimo para cobertura arbórea total seja de $30 \%$ do território do Estado (Melo e Faria, 2004), correspondente às exigências mínimas da legislação vigente (áreas de preservação permanente e reservas legais), ainda restariam cerca de 3,1 milhões de hectares a serem reflorestados, aproximadamente $12,5 \%$ de toda a extensão do Estado de São Paulo. Esses números se aproximam daqueles apresentados por Castanho Filho et al. (2009), que afirmam ser necessário reflorestar entre 1,7 e 3 milhões de hectares, entre plantios de essências nativas e exóticas, para que as necessidades gerais de conservação, preservação, manutenção, utilização sustentada e restauração da cobertura vegetal sejam adequadamente supridas.

Além de suprir a necessidade de florestas destinadas à proteção ambiental, a silvicultura deve também atender à demanda de madeira. No Brasil, o extrativismo de árvores nativas corresponde a aproximadamente $18,6 \%$ do total de madeira produzida no país (IBGE, 2013). Ainda que esse seja o mais baixo patamar, considerando a última década, tal número ainda pode sofrer variações, uma vez que estimativas apontam que a demanda por madeira no Brasil, atualmente girando em torno de 15 milhões de $\mathrm{m}^{3}$.ano ${ }^{-1}$, pode ultrapassar os 20 milhões de $\mathrm{m}^{3}$.ano ${ }^{-1}$ até 2020 (Serviço Florestal Brasileiro - SFB; Instituto de Pesquisa Ambiental da Amazônia - IPAM, 2011). Esse crescimento na demanda certamente aumentará ainda mais a pressão da indústria madeireira sobre os remanescentes nativos (Romeiro et al., 2004).

O desafio da recuperação de florestas em áreas tão extensas esbarra na necessidade de unir os proprietários de terra e a indústria sob a supervisão e estímulo do governo. Mais do que isso, também é preciso demonstrar aos produtores rurais o potencial da silvicultura sustentável como alternativa às demais atividades agropecuárias (Bacha, 2008). 
A silvicultura brasileira tem-se baseado em espécies exóticas dos gêneros Eucalyptus e Pinus, que atualmente correspondem a mais de 92\% da madeira produzida para fins de exploração (Indústria Brasileira de Árvores - IBA, 2015). Dessa maneira, a demanda por madeira nativa para nichos do mercado, tais como a indústria moveleira e a de construção civil, tem sido suprida pela extração não sustentável de florestas naturais (Machado e Bacha, 2002). A silvicultura da maior parte das essências nativas brasileiras nunca foi objeto de experimentação como deveria e esta lacuna, agravada pela deficiência também na disseminação do conhecimento, dificulta a adoção de medidas extensivas que possam estimular e favorecer novos empreendimentos (Brasil, 2007). No cenário atual, em que a legislação impõe a silvicultura de espécies nativas em plantios mistos como a principal alternativa de exploração econômica para as áreas de reserva legal, estudos sobre o cultivo destas espécies ganham importância não só pelos produtos diferenciados, mas também pela extensão da demanda.

Já é conhecido que o desenvolvimento de espécies vegetais em plantios consorciados é influenciado por atributos das espécies em si, tais como o seu porte ou ritmo natural de crescimento (Kageyama e Castro, 1989). Porém, além de depender de seus atributos intrínsecos, o crescimento de uma espécie arbórea em povoamentos mistos, naturais ou plantados, depende de suas adaptações para competir com as demais espécies pelos recursos do meio, especialmente luz, água do solo e nutrientes (Gerhardt, 1996; Balderrama e Chazdon, 2005; Poorter e Markesteijn, 2008). O plantio consorciado de espécies de diferentes estágios sucessionais, que visa melhorar a produtividade madeireira baseado nos princípios da "produção competitiva" e da "facilitação" (Vandermeer, 1989; Kelty, 2006), ainda beneficia o ambiente ao criar sistemas cujos processos ecológicos são mais semelhantes aos de uma floresta natural quando comparados aos plantios monoespecíficos (Piotto, 2008). Além do benefício ecológico, os plantios mistos trazem ainda benefícios sociais, uma vez que fixam o homem ao campo devido à demanda por mão de obra, ausência de sazonalidade no cultivo, flexibilidade de comercialização e diversidade da produção (Kleinpaul et al., 2010).
O presente trabalho objetivou comparar a sobrevivência e o crescimento de duas espécies arbóreas tropicais - $\mathrm{o}$ angico vermelho (Anadenanthera colubrina var. cebil (Griseb.) Altschul) e a aroeira (Myracrodruon urundeuva Allemão), ambas de alto valor comercial como produtoras de madeira, em consorciação com a espécie pioneira capixingui (Croton floribundus Spreng.), espécie pioneira comum na região do estudo, em diferentes proporções entre as espécies plantadas. A consorciação de espécies não pioneiras com espécies pioneiras de rápido crescimento tem sido recomendada em plantios heterogêneos com espécies nativas, especialmente visando reduzir operações de manutenção e melhorar a forma das espécies de interesse comercial. No contexto das complexas interações entre espécies em povoamentos mistos, testaram-se as seguintes hipóteses: 1) diferentes proporções no plantio das espécies resultariam em diferenças no seu ritmo de crescimento, e 2) a resposta mediante o consórcio com a espécie pioneira seria diferente entre as duas espécies analisadas. Esperava-se que a consorciação beneficiasse ambas as espécies e que houvesse uma proporção ótima entre a espécie-alvo e a espécie pioneira, resultando em melhor crescimento para cada uma das espécies analisadas.

\section{MATERIAL E MÉTODOS}

\subsection{Local}

$\mathrm{O}$ estudo foi realizado em dois experimentos de restauração florestal instalados no ano de 1997, na microbacia hidrográfica do ribeirão Água da Cachoeira, tributário da bacia hidrográfica do Médio Paranapanema, município de Paraguaçu Paulista - SP, localizada entre as coordenadas $50^{\circ} 34^{\prime} 22^{\prime \prime}$ a $50^{\circ} 37^{\prime} 17^{\prime \prime} \mathrm{W}$, e $22^{\circ} 17^{\prime} 36^{\prime \prime}$ a $22^{\circ} 23^{\prime} 10$ 'S, a uma altitude aproximada de $510 \mathrm{~m}$ (Melo e Faria, 2004). O solo das áreas experimentais é classificado como Argissolo vermelho-amarelo distrófico abrúptico, que se caracteriza pela baixa fertilidade e pelo horizonte $\mathrm{B}$ textural, que dificulta a infiltração de água, tornando-o suscetível à erosão e acentuando a deficiência hídrica em períodos de estiagem (Campos e Cardoso, 2004). 
O clima local é do tipo Cfa, tropical úmido sem estação seca, segundo a classificação de Köppen (Melo e Faria, 2004), com precipitação anual entre 1.430 e 1.517 mm (Fritzsons et al., 2012). A vegetação existente na região, anteriormente às perturbações, consistia em um mosaico de Cerrado e Floresta Estacional Semidecidual, tal como em toda a bacia hidrográfica do Médio Paranapanema (Melo e Faria, 2004). A área experimental foi utilizada para agricultura e pastagem durante várias décadas, não havendo potencial de regeneração natural.

\subsection{Espécies Utilizadas}

Nos experimentos foram utilizadas três espécies florestais nativas da região:

- Anadenanthera colubrina var. cebil (Griseb.) Altschul: conhecida popularmente como angico-vermelho, pertence à família Fabaceae. Árvore semicaducifólia, de crescimento moderado a rápido, pode atingir até $30 \mathrm{~m}$ de altura e $120 \mathrm{~cm}$ de diâmetro à altura do peito - DAP, apresentando maior porte em florestas estacionais do que em fisionomias abertas. Considerada espécie secundária inicial, o angico-vermelho apresenta características de planta heliófila, demonstra média tolerância a baixas temperaturas e é fixadora de nitrogênio por meio de associação com bactérias do solo. Pode ser cultivada em plantio homogêneo ou em consórcio com espécie pioneira para melhorar a forma do fuste. Por ser uma espécie calcífila, desenvolve-se melhor em solos com boa oferta de cálcio e pH elevado (Carvalho, 1994). A madeira apresenta alta densidade (média entre 0,84 g.cm ${ }^{-3}$ e $1,10 \mathrm{~g} . \mathrm{cm}^{-3}$ ), alta durabilidade e alta resistência ao apodrecimento (Rocha et al., 2000). É recomendada para construção rural, naval e civil, podendo ser também utilizada na fabricação de móveis e carvão;

- Myracrodruon urundeuva Allemão: popularmente denominada aroeira ou urundeúva, pertence à família Anacardiaceae. Árvore caducifólia, de crescimento lento, sua altura varia de 5 a $30 \mathrm{~m}$ e sua diâmetro à altura do peito - DAP varia entre 30 e $60 \mathrm{~cm}$, dependendo das características do local de cultivo. Sua classificação sucessional ainda é discutida entre autores, sendo considerada espécie secundária inicial, secundária tardia e também climácica exigente de luz.
Sobrevive ao fogo, tornando-se frequente em vegetação secundária através de reprodução vegetativa. Espécie heliófila de média tolerância a baixas temperaturas. Em condições de cultivo, geralmente apresenta péssima forma, criando ramificações e bifurcando-se cerca de 2 a $3 \mathrm{~m}$ do solo, necessitando de ação artificial para criar um fuste adequado para a exploração silvicultural (Carvalho, 1994). Espécie calcífila, apresenta melhor desenvolvimento em solos com características calcárias e pH elevado (Venturin et al., 2000). Apresenta madeira densa, variando entre 1 a $1,21 \mathrm{~g} . \mathrm{cm}^{-3}$, com cerne altamente durável e imputrescível, sendo considerada a madeira mais resistente do Brasil (Carvalho, 2003). É recomendada para a construção externa e civil, sendo inadequada para a produção de celulose e papel. Considerada vulnerável à extinção em todas as áreas de ocorrência no Brasil (Nunes et al., 2008);

- Croton floribundus Spreng.: popularmente conhecida como capixingui, pertence à família Euphorbiaceae. Espécie semicaducifólia, de crescimento rápido, atinge até $20 \mathrm{~m}$ de altura e $60 \mathrm{~cm}$ de DAP quando adulta. Pioneira, heliófila, intolerante a baixas temperaturas, sendo típica em Floresta Estacional Semidecidual. Sua madeira é moderadamente densa $(0,5$ a $\left.0,6 \mathrm{~g} \cdot \mathrm{cm}^{-3}\right)$ e muito suscetível ao apodrecimento, apresentando aplicação restrita (Carvalho, 1994).

\subsection{Desenho Experimental}

Este estudo se baseou em dados coletados em dois plantios experimentais distintos, espacialmente próximos dentro de uma mesma microbacia, sendo um para a consorciação de Anadenanthera colubrina var. cebil e Croton floribundus (ensaio I) e o outro entre Myracrodruon urundeuva e Croton floribundus (ensaio II). Nos dois plantios experimentais, adotou-se o delineamento em blocos casualizados, com cinco repetições de quatro tratamentos para cada uma das espécies-alvo. Por sua vez, os tratamentos foram compostos, respectivamente, por $75 \%, 50 \%$, $25 \%$ e $15 \%$ de indivíduos da espécie-alvo e completados por indivíduos da espécie pioneira, obtendo-se as proporções pela distribuição sistemática das mudas dentro de cada linha, alternando-se as espécies dentro da linha de acordo com as proporções dos diferentes tratamentos. 
Cada parcela útil foi constituída por quatro linhas contendo oito árvores cada (32 plantas). Entre as parcelas foi mantida bordadura simples, o espaçamento entre mudas foi de $3 \mathrm{~m} \times 2 \mathrm{~m}$ para ambos os ensaios, de modo que a área de cada parcela foi de $192 \quad \mathrm{~m}^{2}$.

As mudas utilizadas nos experimentos foram produzidas em viveiro, em embalagens plásticas de $800 \mathrm{~mL}$, indo ao campo com idade aproximada de seis meses. As sementes utilizadas foram coletadas na região de estudo pela equipe do Instituto Florestal. Não foi efetuada correção dos solos e nem aplicação de fertilizantes e a manutenção das mudas (capina, coroamento e controle de formigas cortadeiras) foi realizada por dois anos após o plantio. Nenhuma operação de manejo, como desbaste ou desrama, foi realizada durante todo o experimento, de modo que o crescimento das árvores foi condicionado pelas relações de competição estabelecidas com a densidade de plantio (1.667 mudas por hectare).

\subsection{Coleta e Análise de Dados}

Os dados foram coletados no período de dezembro/2011 a janeiro/2012, de modo que as árvores plantadas tinham idade de 15 anos. O DAP (tomado a 1,30 m do solo) de cada tronco foi medido com suta. A altura total da árvore foi estimada visualmente, com treinamento (Silva et al., 2012). A partir dos dados de diâmetro, foi obtida a área basal de cada indivíduo e o volume foi calculado segundo a equação do cone, utilizando-se a altura estimada de cada árvore.

Com base nos dados obtivemos, para cada espécie dentro de cada parcela, informações sobre sobrevivência (\%), altura média, incremento médio anual em altura (dividindo-se a altura total pela idade), diâmetro equivalente $-d g$ (diâmetro único estimado a partir da área basal do indivíduo, resultante da soma das áreas basais de todos os fustes, para os indivíduos que apresentaram múltiplos fustes) e incremento médio anual em $d g$ (dividindo o $d g$ aos 15 anos pela idade). Considerando-se a proporção entre espécies em cada tratamento, o espaçamento de plantio e a área de cada parcela, foi estimado o volume de madeira de cada espécie por hectare, bem como o volume total de madeira em cada um dos sistemas de consórcio.
Para as análises estatísticas, os dados de porcentagens de sobrevivência foram transformados em arcsen raiz $(\mathrm{x} / 100)$. Após verificação dos pressupostos de normalidade e homocedasticidade das variâncias, todos os dados obtidos foram submetidos à análise de variância - ANOVA pelo teste $\mathrm{F}$ e as médias comparadas pelo teste de Tukey a 5\% por meio do software BioEstat 5.3.

\section{RESULTADOS}

$\mathrm{Na}$ consorciação entre Myracrodruon urundeuva e Croton floribundus, foram observadas diferenças entre os tratamentos em sobrevivência, crescimento em altura e diâmetro de cada uma das espécies consorciadas, exceto no caso da sobrevivência de C. floribundus, que variou entre 63,5 e $74,5 \%$ e não diferiu entre os tratamentos $\left(\mathrm{F}_{(3,16)}=0,4119 ; \mathrm{p}=0,7495 ; \alpha=0,05\right)$ (Tabela 1). Para $M$. urundeuva a sobrevivência foi alta em todos os tratamentos (mínimo de 93,1\%), sendo menor quando a proporção da espécie foi mais elevada ( $75 \%$ das mudas), em comparação com os demais tratamentos $\left(\mathrm{F}_{(3,16)}=3,2748 ; \mathrm{p}=0,0479 ; \alpha=0,05\right)$. A mortalidade diferenciada entre as espécies resultou em uma densidade final do sistema inversamente proporcional à proporção de pioneiras plantadas.

A altura média das árvores foi baixa para ambas as espécies, não ultrapassando $6,18 \mathrm{~m}$ em 15 anos (Tabela 1). O crescimento em altura foi ainda menor, no caso da M. urundeuva, quando a proporção da espécie pioneira foi baixa (25\%), em comparação com os demais tratamentos $\left(\mathrm{F}_{(3,250)}=5,5031 ; \mathrm{p}=0,0015 ; \alpha=0,05\right)$. Por outro lado, o crescimento das árvores de C. floribundus foi menor quando a espécie foi plantada em alta proporção (75\%), em comparação com o plantio em baixa proporção $(25 \%)$ $\left(\mathrm{F}_{(3,266)}=3,5639 ; \mathrm{p}=0,0147 ; \alpha=0,05\right)$.

Para o crescimento em diâmetro, o comportamento das espécies seguiu o verificado para a altura. As árvores de $M$. urundeuva cresceram mais lentamente quando a proporção da espécie pioneira foi baixa (25\%) do que sob alta proporção da espécie pioneira $\left(\mathrm{F}_{(3,251)}=5,3455\right.$; $\mathrm{p}=0,0018 ; \alpha=0,05)$. Em contrapartida, as árvores de C. floribundus cresceram muito mais em diâmetro quando plantadas em baixa proporção $(25 \%)$ do que nas demais proporções $\left(\mathrm{F}_{(3,266)}=9,4254\right.$; $\mathrm{p}<0,0001 ; \alpha=0,05)$. 
SOUZA, C.E.M. de; VILAS BÔAS, O.; DURIGAN, G. Sobrevivência e crescimento de espécies tropicais madeireiras

Tabela 1. Sobrevivência e crescimento de Myracrodruon urundeuva e Croton floribundus (espécie pioneira - P) aos 15 anos após o plantio em consórcio em diferentes proporções. IMA altura - incremento médio anual em altura, $\mathrm{dg}$ - diâmetro equivalente, IMA dg - incremento médio anual em dg.

Table 1. Survival and growth of Myracrodruon urundeuva and Croton floribundus (pioneer species - P) 15 years after intercropping on different proportions. AAI height - average annual increment in height, dg - equivalent diameter, AAI $\mathrm{dg}$ - average annual increment in $\mathrm{dg}$.

\begin{tabular}{|c|c|c|c|c|c|c|}
\hline Espécie & Tratamento & Sobrevivência (\%) & Altura (m) & $\begin{array}{l}\text { IMA altura } \\
\left(\mathrm{m} . \mathrm{ano}^{-1}\right)\end{array}$ & $\operatorname{dg}(\mathrm{cm})$ & $\begin{array}{c}\text { IMA dg } \\
\left(\mathrm{cm}^{2} \cdot \text { ano }^{-1}\right)\end{array}$ \\
\hline \multirow[t]{4}{*}{ M. urundeuva } & $\mathrm{A}(25 \% \mathrm{P})$ & $93,1 b$ & $3,97 b$ & $0,26 b$ & $3,80 \mathrm{~b}$ & $0,25 b$ \\
\hline & $\mathrm{B}(50 \% \mathrm{P})$ & $100 \mathrm{a}$ & $5,02 \mathrm{a}$ & $0,33 \mathrm{a}$ & $4,89 \mathrm{ab}$ & $0,36 a b$ \\
\hline & $\mathrm{C}(75 \% \mathrm{P})$ & $99,5 \mathrm{a}$ & $5,53 \mathrm{a}$ & $0,36 \mathrm{a}$ & $5,89 \mathrm{a}$ & $0,39 \mathrm{a}$ \\
\hline & $\mathrm{D}(85 \% \mathrm{P})$ & $99,1 \mathrm{a}$ & $5,40 \mathrm{ab}$ & $0,34 a b$ & $6,12 \mathrm{a}$ & $0,41 \mathrm{a}$ \\
\hline \multirow[t]{4}{*}{ C. floribundus } & $\mathrm{A}(25 \% \mathrm{P})$ & $63,5 \mathrm{a}$ & $6,18 \mathrm{a}$ & $0,41 \mathrm{a}$ & $12,33 \mathrm{a}$ & $0,82 \mathrm{a}$ \\
\hline & $\mathrm{B}(50 \% \mathrm{P})$ & $72,5 \mathrm{a}$ & $4,87 \mathrm{ab}$ & $0,32 \mathrm{ab}$ & $7,18 b$ & $0,48 b$ \\
\hline & $\mathrm{C}(75 \% \mathrm{P})$ & $77,5 \mathrm{a}$ & $5,06 a b$ & $0,34 a b$ & $6,95 \mathrm{~b}$ & $0,47 \mathrm{~b}$ \\
\hline & $\mathrm{D}(85 \% \mathrm{P})$ & $74,5 \mathrm{a}$ & $4,28 b$ & $0,29 b$ & $5,89 \mathrm{~b}$ & $0,40 \mathrm{~b}$ \\
\hline
\end{tabular}

Para cada espécie, valores seguidos pela mesma letra dentro de uma coluna não diferem estatisticamente entre si pelo teste de Tukey $(\mathrm{p}>0,05)$.

For each species, values followed by the same letter within a column do not differ statistically by Tukey test ( $\mathrm{p}>0.05)$.

$\mathrm{Na}$ consorciação entre Anandenanthera colubrina var. cebil e Croton floribundus, verifica-se que houve também diferenças na sobrevivência e no crescimento das espécies quando plantadas em diferentes proporções (Tabela 2). Contudo, diferenças nos resultados foram observadas apenas para A. colubrina var. cebil. A sobrevivência desta foi de $100 \%$ quando a espécie pioneira foi plantada em alta densidade, sendo menor nos tratamentos em que a proporção da espécie pioneira foi inferior a $50 \%\left(\mathrm{~F}_{(3,16)}=5,4926 ; \mathrm{p}=0,0088\right.$, $\alpha=0,05)$. Considerando-se que a mortalidade de C. floribundus, cuja média não variou entre tratamentos $\left(\mathrm{F}_{(3,16)}=0,8540 ; \mathrm{p}=0,5130 ; \alpha=0,05\right)$, foi mais que o dobro da mortalidade de A. colubrina var. cebil, depreende-se que a densidade final do sistema foi maior quanto menor a proporção da espécie pioneira, padrão este que também foi observado para M. urundeuva.

$\mathrm{O}$ crescimento das árvores de $A$. colubrina var. cebil em altura foi maior quando $75 \%$ das mudas plantadas eram da espécie pioneira, em comparação com o tratamento com apenas $25 \%$ de pioneiras $\left(\mathrm{F}_{(3,236)}=4,3884 ; \mathrm{p}=0,0053 ; \alpha=0,05\right)$ (Tabela 2). Para o diâmetro, o padrão foi ainda mais consistente, com maior crescimento de A. colubrina var. cebil em diâmetro nos dois tratamentos em que a proporção de pioneiras era mais alta, em comparação com os dois tratamentos com menores proporções da pioneira $\left(\mathrm{F}_{(3,236)}=18,3680 ; \mathrm{p}<0,0001 ; \alpha=0,05\right)$. 
SOUZA, C.E.M. de; VILAS BÔAS, O.; DURIGAN, G. Sobrevivência e crescimento de espécies tropicais madeireiras

Tabela 2. Sobrevivência e crescimento de Anadenanthera colubrina var. cebil e Croton floribundus (espécie pioneira - P) aos 15 anos após o plantio em consórcio em diferentes proporções. IMA altura - incremento médio anual em altura, $\mathrm{dg}$ - diâmetro equivalente, IMA dg - incremento médio anual em $\mathrm{dg}$.

Table 2. Survival and growth of Anadenanthera colubrina var. cebil and Croton floribundus (pioneer species - P) 15 years after intercropping on different proportions. AAI height - average annual increment in height, $\mathrm{dg}$ - equivalent diameter, AAI dg - average annual increment in $\mathrm{dg}$.

\begin{tabular}{|c|c|c|c|c|c|c|}
\hline Espécie & Tratamento & Sobrevivência (\%) & Altura (m) & $\begin{array}{l}\text { IMA altura } \\
\left(\mathrm{m} \cdot \mathrm{ano}^{-1}\right)\end{array}$ & $\mathrm{dg}(\mathrm{cm})$ & $\begin{array}{c}\text { IMA dg } \\
\left(\mathrm{cm}^{\left.-\mathrm{ano}^{-1}\right)}\right.\end{array}$ \\
\hline \multirow[t]{4}{*}{ A. colubrina var. cebil } & $\mathrm{A}(25 \% \mathrm{P})$ & $88,8 \mathrm{~b}$ & $10,91 b$ & $0,73 b$ & $16,99 b$ & $1,13 b$ \\
\hline & $\mathrm{B}(50 \% \mathrm{P})$ & $92,7 b$ & $12,49 \mathrm{ab}$ & $0,83 \mathrm{ab}$ & $20,89 b$ & $1,39 b$ \\
\hline & $\mathrm{C}(75 \% \mathrm{P})$ & $94 \mathrm{ab}$ & $14,13 \mathrm{a}$ & $0,94 \mathrm{a}$ & $29,15 \mathrm{a}$ & $1,94 \mathrm{a}$ \\
\hline & $\mathrm{D}(85 \% \mathrm{P})$ & $100 \mathrm{a}$ & $12,90 \mathrm{ab}$ & $0,86 \mathrm{ab}$ & $30,28 \mathrm{a}$ & $2,02 \mathrm{a}$ \\
\hline \multirow[t]{4}{*}{ C. floribundus } & $\mathrm{A}(25 \% \mathrm{P})$ & $34,2 \mathrm{a}$ & $5,98 \mathrm{a}$ & $0,39 \mathrm{a}$ & $8,05 \mathrm{a}$ & $0,54 \mathrm{a}$ \\
\hline & $\mathrm{B}(50 \% \mathrm{P})$ & $40,6 a$ & $7,43 \mathrm{a}$ & $0,49 a$ & $10,11 \mathrm{a}$ & $0,67 \mathrm{a}$ \\
\hline & $\mathrm{C}(75 \% \mathrm{P})$ & $38,9 a$ & $6,07 \mathrm{a}$ & $0,40 \mathrm{a}$ & $8,50 \mathrm{a}$ & $0,57 \mathrm{a}$ \\
\hline & $\mathrm{D}(85 \% \mathrm{P})$ & $44 a$ & $7,08 \mathrm{a}$ & $0,47 \mathrm{a}$ & $9,55 \mathrm{a}$ & $0,64 a$ \\
\hline
\end{tabular}

Para cada espécie, valores seguidos pela mesma letra dentro de uma coluna não diferem estatisticamente entre si pelo teste de Tukey $(\mathrm{p}>0,05)$.

For each species, values followed by the same letter within a column do not differ statistically by Tukey test ( $\mathrm{p}>0.05$ ).

As comparações de produção de madeira entre os sistemas de consórcio mostram, para ambas as espécies-alvo, que o volume médio de árvores individuais tende a ser maior quanto menor a proporção da espécie com relação à espécie pioneira $\left(M\right.$. urundeuva $-\mathrm{F}_{(3,250)}=5,1771$; $\mathrm{p}=0,0021, \alpha=0,05 ; A$. colubrina var. cebil $\left.\mathrm{F}_{(3,236)}=14,4212 ; \mathrm{p}<0,0001 ; \alpha=0,05\right)$ (Tabela 3). Embora os dois experimentos não tenham sido comparados estatisticamente, considerando-se que as condições ambientais das duas áreas eram muito semelhantes, a diferença no volume total de madeira entre os sistemas indica que $A$. colubrina var. cebil resulta em maior produtividade do que M. urundeuva. Porém, não houve efeito da proporção de pioneiras plantadas sobre o volume total por hectare de nenhuma das duas espécies-alvo (M. urundeuva $-\mathrm{F}_{(3,16)}=0,1791 ; \mathrm{p}=0,9087$; $\alpha=0,05 ;$ A colubrina var. cebil $-\mathrm{F}_{(3,16)}=1,7177$; $\mathrm{p}=0,2028 ; \alpha=0,05)$.

Tabela 3. Parâmetros relacionados à produção de madeira das espécies-alvo (Myracrodruon urundeuva e Anadenanthera colubrina var. cebil) quando consorciadas com espécie pioneira - P (Croton floribundus) nos diferentes tratamentos.

Table 3. Parameters related to timber production of the target species (Myracrodruon urundeuva and Anadenanthera colubrina var. cebil) when intercropped with a pioneer species - P (Croton floribundus) on different treatments.

\begin{tabular}{ccccc}
\hline Espécie & Tratamento & $\begin{array}{c}\text { Vol. médio por } \\
\text { árvore }\left(\mathrm{m}^{3}\right)\end{array}$ & $\begin{array}{c}\text { Vol. de madeira } \\
\text { espécie-alvo }\left(\mathrm{m}^{3} . \mathrm{ha}^{-1}\right)\end{array}$ & $\begin{array}{c}\text { Vol. total de madeira } \\
\text { no consórcio }\left(\mathrm{m}^{3} \cdot \mathrm{ha}^{-1}\right)\end{array}$ \\
\hline M. urundeuva & $\mathrm{A}(25 \% \mathrm{P})$ & $0,0041 \mathrm{~b}$ & $4,74 \mathrm{a}$ & $16,26 \mathrm{a}$ \\
& $\mathrm{B}(50 \% \mathrm{P})$ & $0,0064 \mathrm{ab}$ & $5,31 \mathrm{a}$ & $12,94 \mathrm{a}$ \\
& $\mathrm{C}(75 \% \mathrm{P})$ & $0,0105 \mathrm{a}$ & $4,26 \mathrm{a}$ & $18,46 \mathrm{a}$ \\
& $\mathrm{D}(85 \% \mathrm{P})$ & $0,0136 \mathrm{a}$ & $3,41 \mathrm{a}$ & $15,83 \mathrm{a}$ \\
\hline A. colubrina var. cebil & $\mathrm{A}(25 \% \mathrm{P})$ & $0,1542 \mathrm{~b}$ & $167,08 \mathrm{a}$ & $169,32 \mathrm{a}$ \\
& $\mathrm{B}(50 \% \mathrm{P})$ & $0,2240 \mathrm{~b}$ & $168,03 \mathrm{a}$ & $177,96 \mathrm{a}$ \\
& $\mathrm{C}(75 \% \mathrm{P})$ & $0,4139 \mathrm{a}$ & $155,22 \mathrm{a}$ & $166,37 \mathrm{a}$ \\
& $\mathrm{D}(85 \% \mathrm{P})$ & $0,4083 \mathrm{a}$ & $106,34 \mathrm{a}$ & $122,24 \mathrm{a}$ \\
\hline
\end{tabular}

Para cada espécie, valores seguidos pela mesma letra dentro de uma coluna não diferem estatisticamente entre si pelo teste de Tukey $(\mathrm{p}>0,05)$.

For each species, values followed by the same letter within a column do not differ statistically by Tukey test ( $\mathrm{p}>0.05)$. 


\section{DISCUSSÃO}

O incremento anual em diâmetro de Myracrodruon urundeuva nos plantios consorciados neste estudo (máximo de $0,41 \mathrm{~cm}^{-a n o}{ }^{-1}$ ) foi inferior ao que tem sido mencionado na literatura para plantios puros desta espécie, com valores geralmente superiores a 0,5 cm.ano ${ }^{-1}$ (Garrido, 1981; Barros, 1970; Carvalho, 2003). O incremento médio anual em diâmetro para Croton floribundus (máximo de $0,82 \mathrm{~cm}$.ano ${ }^{-1}$ ) também foi inferior ao mencionado na literatura para a espécie, mesmo em plantios heterogêneos, que costuma ser superior a $2 \mathrm{~cm}^{2}$ ano ${ }^{-1}$ (Asperti, 2001; Santarelli, 1990; Speltz, 1968). Tais resultados poderiam estar relacionados com a baixa fertilidade dos solos nas áreas experimentais, desfavorecendo o crescimento das espécies plantadas; porém, $\mathrm{o}$ fato de que todas as espécies ocorrem naturalmente na região do estudo, associado ao bom desempenho comparativo de Anadenanthera colubrina var. cebil, levam à consideração de que a falta de desbastes ao longo dos 15 anos, desde o plantio, é a explicação mais provável para as baixas taxas de incremento em diâmetro. Essa condição deve ter submetido as plantas a alto nível de competição por longo tempo em todos os tratamentos, especialmente por água do solo, resultando em crescimento lento; exceto para A. colubrina var. cebil, espécie fixadora de nitrogênio que parece ser dominante e que apresenta elevada plasticidade fenotípica (Toledo Filho, 1988), levando aparente vantagem competitiva com relação a C. floribundus. A inexistência de diferenças no crescimento das espécies entre os tratamentos, nesses mesmos experimentos, quando avaliados aos cinco anos de idade (Melo e Faria, 2004), é um indício a mais de que os diferentes níveis de competição impostos pelas diferentes proporções de espécies pioneiras, ao longo do tempo, foram um fator-chave para influenciar o crescimento das árvores plantadas.

Os sistemas silviculturais analisados apresentaram alguns padrões. A mortalidade da espécie pioneira foi superior à das espécies-alvo, o que é esperado com base no conhecimento sobre a longevidade das espécies de diferentes grupos sucessionais (Budowsky, 1965; Denslow, 1980; Kageyama e Gandara, 2001). Outro padrão encontrado neste estudo, inesperado à primeira vista, foram maiores valores de diâmetro das espécies-alvo nos tratamentos com maior densidade de plantio da espécie pioneira. Esse resultado parece conflitar com as teorias existentes, pois era de se esperar que as espécies pioneiras, de rápido crescimento, exercessem maior competição quanto maior sua proporção nos sistemas, prejudicando o crescimento das espécies não pioneiras (Durigan et al., 1999; Laurance et al., 2006; Poorter et al., 2010, Tabarelli et al., 2010); porém, a análise conjunta dos resultados de crescimento e sobrevivência leva à constatação de que as árvores das espécies-alvo cresceram melhor quanto menor a densidade de árvores existentes no sistema aos 15 anos, o que é coerente com todo o conhecimento já existente sobre silvicultura e competição (Amoroso e Turnblom, 2006; Kelty, 2006). Na consorciação de M. urundeuva com C. floribundus, aos 15 anos, o tratamento com $25 \%$ de pioneiras tinha, no total, 126 árvores a mais por hectare do que o tratamento com $85 \%$ de pioneiras. Na consorciação de A. colubrina var. cebil com C. floribundus, o tratamento com $25 \%$ de pioneiras tinha no total 379 árvores a mais por hectare do que o tratamento com $85 \%$ de pioneiras, tendo diminuído drasticamente a proporção de árvores da espécie pioneira em relação à proporção em que foram plantadas. Portanto, a morte das árvores pioneiras funcionou, provavelmente, como uma operação de desbaste, reduzindo a competição por recursos do meio e, assim, beneficiando as árvores da espécie-alvo em ambos os casos - de M. urundeuva e de A. colubrina var. cebil.

Embora as árvores das espécies-alvo tenham crescido mais nos sistemas com maior proporção de pioneiras, a produção em volume de madeira por hectare não diferiu entre tratamentos para nenhuma das duas espécies, havendo um trade-off entre crescimento $\mathrm{e}$ densidade, decorrente da competição, já que não houve desbaste. Os resultados analisados para ambos os ensaios apontam para a necessidade de manejo dos sistemas, que deve ser distinta entre as diferentes combinações de espécies, considerando as diferenças no ritmo de crescimento das espécies-alvo. $\mathrm{O}$ desbaste da espécie pioneira poderia ter resultado em melhor crescimento das espécies-alvo em ambas as combinações de espécies; porém, o momento ideal do desbaste e a resposta das espécies-alvo quanto ao seu crescimento e produção de madeira não parece seguir um padrão, e isto se deve ao ritmo de crescimento das espécies. 
O volume total de madeira por hectare foi surpreendentemente distinto entre os dois ensaios. Embora se tratem de experimentos diferentes, o crescimento médio em dg semelhante das árvores de C. floribundus nos dois casos (8 e $9 \mathrm{~cm}$ em 15 anos na consorciação com $M$. urundeuva e $A$. colubrina var. cebil, respectivamente) leva a crer que as condições ambientais sejam semelhantes nas duas áreas experimentais, que são bastante próximas, de modo que a capacidade de suporte do meio deve ser semelhante; porém, ainda que os dois ensaios não tenham sido comparados estatisticamente, o volume total de madeira por hectare para o experimento como um todo, na combinação de A. colubrina var. cebil com C. floribundus, foi dez vezes maior em relação à combinação de M. urundeuva com C. floribundus. Essa diferença está relacionada com a eficiência das espécies em utilizar os recursos do meio, como nutrientes e, especialmente, água, que é um recurso escasso na região, que tem clima estacional. A grande diferença de produtividade entre os sistemas não deve ser negligenciada, já que esta desproporção se mantém mesmo quando apenas o volume de madeira das espécies-alvo é considerado. Conclui-se que em plantios consorciados, quando o crescimento lento de uma espécie poderia, teoricamente, ser compensado pelo crescimento da(s) outra(s) espécies, gerando florestas com biomassa ou produtividade semelhantes para condições ambientais semelhantes, isto, de fato, não acontece na prática. Assim, na silvicultura de espécies nativas, a produtividade do sistema continua dependendo da escolha de espécies, mesmo em plantios mistos.

\section{CONCLUSÕES}

A hipótese de que diferentes proporções das espécies na ocasião do plantio resultariam em diferenças no seu ritmo de crescimento foi confirmada neste estudo. $\mathrm{O}$ crescimento individual das árvores das duas espécies-alvo foi maior quanto maior a proporção de árvores da espécie pioneira no consórcio, porém, o resultado, que parece apontar um benefício da espécie pioneira para a não pioneira, na verdade é explicado pela maior mortalidade nos sistemas com mais árvores pioneiras, reduzindo a competição e, indiretamente, favorecendo o desenvolvimento das espécies-alvo.
A hipótese de que a resposta mediante o consórcio com a espécie pioneira seria diferente entre as duas espécies analisadas foi comprovada no que tange à produção de madeira. Não foi encontrado, porém, para nenhuma das duas espécies, a proporção ótima entre a espécie-alvo e a espécie pioneira, que resultasse em melhor crescimento das árvores individuais e maior volume de madeira por hectare.

\section{AGRADECIMENTOS}

Os autores agradecem a Silvio dos Santos e demais funcionários da Estação Experimental J. J. Galhardo pela ajuda na coleta dos dados, à Japan International Cooperation Agency pelo financiamento da instalação dos experimentos, aos revisores do periódico pelas sugestões e Giselda Durigan agradece ao $\mathrm{CNPq}$ pela bolsa de Produtividade em Pesquisa (Processo: 302939/2009-1).

\section{REFERÊNCIAS BIBLIOGRÁFICAS}

AMOROSO, M.M.; TURNBLOM, E.C. Comparing productivity of pure and mixed Douglas-fir and western hemlock plantations in the Pacific Northwest. Canadian Journal of Forest Research, v. 36, p. 1484-1496, 2006.

ASPERTI, L.M. Monitoramento e avaliação de um repovoamento florestal implantado com espécies nativas no entorno de uma várzea em Santa Cruz das Palmeiras (SP). 2001. 84 f. Dissertação (Mestrado) - Universidade de Guarulhos, Guarulhos.

BACHA, C.J.C. Análise da evolução do reflorestamento no Brasil. Revista de Economia Agrícola, v. 55, n. 2, p. 5-24, 2008.

BALDERRAMA, S.I.V.; CHAZDON, R.L. Light-dependent seedling survival and growth of four tree species in Costa Rican second-growth rain forests. Journal of Tropical Ecology, v. 21, p. 383-395, 2005.

BARROS, D.P. Ensaio de espaçamento inicial para "aroeira". Silvicultura em São Paulo, v. 7, p. 39-41, 1970. 
BRASIL. Ministério do Meio Ambiente - MMA. Plano Nacional de Silvicultura com Espécies Nativas e Sistemas Agroflorestais - PENSAF. Brasília, DF, 2007. 44 p.

BUDOWSKY, G. Distribution of tropical American rain forest in the light of successional processes. Turrialba, v. 15, n. 1, p. 40-42, 1965.

CAMPOS, S.P.; CARDOSO, L.G. Geoprocessamento aplicado ao planejamento do uso do solo em bacia hidrográfica. In: VILAS BÔAS, O.; DURIGAN, G. Pesquisas em conservação e recuperação ambiental no Oeste Paulista - resultados da Cooperação Brasil/Japão. São Paulo: Páginas \& Letras, 2004. p. 67-79.

CARVALHO, P.E.R. Espécies florestais brasileiras: recomendações silviculturais, potencialidades e uso da madeira. Colombo: EMBRAPA-CNPF; Brasília, DF: EMBRAPA- SPI, 1994. 639 p.

Aroeira verdadeira. Colombo: EMBRAPA Florestas, 2003. 16 p. (Circular Técnica, 82).

CASTANHO FILHO, E.P. et al. Avaliação do Plano de Desenvolvimento Florestal Sustentável - PDFS - 1993/2009. São Paulo: Instituto de Economia Agrícola - IEA, 2009. 52 p.

DENSLOW, J.S. Gap partioning among tropical rain forest trees. Biotropica, v. 12, n. 2, p. 47-55, 1980.

DURIGAN, G.; GURGEL-GARRIDO, L.M.A.; GARRIDO, M.A.O. Desenvolvimento de Plathymenia reticulata Benth. em plantio puro e em consorciação com espécies de diferentes estádios sucessionais. Revista do Instituto Florestal, v. 11, n. 2, p. 131-136, 1999.

FRITZSONS, E. et al. Zoneamento climático para plantio experimental de Pinus maximinoi no Estado de São Paulo. Pesquisa Florestal Brasileira, v. 32, n. 69 , p. 79-92, 2012.

GARRIDO, M.A.O. Caracteres silviculturais e conteúdo de nutrientes no folhedo de alguns povoamentos puros e mistos de espécies nativas. 1981. 105 f. Dissertação (Mestrado) - Universidade de São Paulo, Escola Superior de Agricultura "Luiz de Queiroz", Piracicaba.
GERHARDT, K. Effects of root competition and canopy openness on survival and growth of tree seedlings in a tropical seasonal dry forest. Forest Ecology and Management, v. 82, p. 33-48, 1996.

INDÚSTRIA BRASILEIRA DE ÁRVORES - IBÁ. Relatório Ibá 2015. Brasília, DF, 2015. 80 p.

INSTITUTO BRASILEIRO DE GEOGRAFIA E ESTATÍSTICA - IBGE. Produção da extração vegetal e silvicultura 2013. Rio de Janeiro, 2013. v. 28,66 p.

. Área Territorial Oficial 2013. Rio de Janeiro, 2014. Disponível em: <http://www.ibge.gov.br/home/ geociencias/cartografia/default_territ_area.shtm $>$. Acesso em: 15 jul. 2015.

KAGEYAMA, P.; CASTRO, C.F.A. Sucessão secundária, estrutura genética e plantações de espécies arbóreas nativas. Scientia Forestalis, v. 41/42, p. 83-93, 1989.

.; GANDARA, F.B. Recuperação de áreas ciliares. In: RODRIGUES, R.R.; LEITÃO FILHO, H.F. Matas ciliares: conservação e recuperação. São Paulo: EDUSP: FAPESP, 2001. p. 249-269.

KELTY, M.J. The role of species mixtures in plantation forestry. Forest Ecology and Management, v. 233, p. 195-204, 2006.

KLEINPAUL, I.S. et al. Plantio misto de Eucalyptus urograndis e Acacia mearnsii em sistema agroflorestal: I - produção de biomassa. Ciência Florestal, v. 20, n. 4, p. 621-627, 2010.

LAURANCE, W.F. et al. Rain forest fragmentation and the proliferation of successional trees. Ecology, v. 87, n. 2, p. 469-482, 2006.

MACHADO, J.A.R.; BACHA, C.J.C. Análise da rentabilidade econômica dos reflorestamentos com essências nativas brasileiras: o caso do Estado de São Paulo. Revista de Economia e Sociologia Rural, v. 40, n. 3, p. 581-604, 2002. 
MELO, A.C.G; FARIA, H.H. Três ensaios de crescimento para espécies arbóreas de valor comercial em plantio consorciado a espécie pioneira em Paraguaçu Paulista, SP. In: VILAS BÔAS, O.; DURIGAN, G. (Org.). Pesquisas em conservação e recuperação ambiental no oeste paulista - resultados da Cooperação Brasil/Japão. São Paulo: Páginas \& Letras, 2004. p. 397-407.

NUNES, Y.R.F. et al. Aspectos ecológicos da aroeira (Myracrodruon urundeuva Allemão Anacardiaceae): fenologia e germinação de sementes. Revista Árvore, v. 32, n. 2, p. 233-243, 2008.

PIOTTO, D. A meta-analysis comparing tree growth in monocultures and mixed plantations. Forest Ecology and Management, v. 255, n. 3-4, p. 781-786, 2008.

POORTER, L; MARKESTEIJN, L. Seedling traits determine drought tolerance of tropical tree species. Biotropica, v. 40, n. 3, p. 321-331, 2008.

et al. The importance of wood traits and hydraulic conductance for the performance and life history strategies of 42 rainforest tree species. New Phytologist, v. 185, n. 2, p. 481-492, 2010.

ROCHA, F.T. et al. Durabilidade natural de madeiras em contato com o solo: $\mathrm{V}$ - avaliação final (20 anos). Revista do Instituto Florestal, v. 12, n. 1, p. 59-66, 2000.

ROMEIRO, A.R.; MANGABEIRA, J.A.C; VALLADARES, G.S. Biodiversidade, reflorestamento e agropecuária no Brasil. Florestar Estatístico, v. 7, n. 16, p. 15-22, 2004.

SANTARELLI, E.G. Comportamento de algumas espécies vegetais na recomposição de matas nativas. In: CONGRESSO FLORESTAL BRASILEIRO, 6., 1990, Campos do Jordão. Anais... São Paulo: SBS/SBEF, 1990. p. 232-235.

SÃO PAULO (Estado). Secretaria de Agricultura e Abastecimento - SAA. LUPA - Levantamento Censitário das Unidades de Produção Agropecuária do Estado de São Paulo: dados consolidados do estado de São Paulo 2007/2008. São Paulo: Coordenadoria de Assistência Técnica - CATI, 2008. 24 p.
SERVIÇO FLORESTAL BRASILEIRO - SFB; INSTITUTO DE PESQUISA AMBIENTAL DA AMAZÔNIA - IPAM. Relatório técnico - florestas nativas de produção brasileiras. Brasília, DF, 2011. $28 \mathrm{p}$.

SILVA, G.F. et al. Avaliação de métodos de medição de altura em florestas naturais. Revista Árvore, v. 36, n. 2, p. 341-348, 2012.

SISTEMA DE INFORMAÇÕES FLORESTAIS DO ESTADO DE SÃO PAULO - SIFESP. Quantificação da vegetação natural remanescente para os municípios do estado de São Paulo. Disponível em: <http://www.iflorestal.sp.gov.br/ sifesp/tabelas/municipio_maior_porc.pdf $>$. Acesso em: 15 out. 2015.

SPELTZ, R.M. Comportamento de algumas essências nativas na Fazenda Monte Alegre. In: CONGRESSO FLORESTAL BRASILEIRO, 1968, Curitiba. Anais... Curitiba: Federação das Indústrias do Estado do Paraná; Associação Paranaense de Engenheiros Florestais, 1968. p. 299-302.

TABARELLI, M. et al. Effects of pioneer tree species hyperabundance on forest fragments in northeastern Brazil. Conservation Biology, v. 24, n. 6, p. 1654-63, 2010.

TOLEDO FILHO, D.V. Competição de espécies arbóreas de cerrado. Boletim Técnico do Instituto Florestal, v. 42, p. 61-70, 1988.

VANDERMEER, J. Ecology of intercropping. Cambridge: Cambridge University Press, 1989. $237 \mathrm{p}$.

VENTURIN, R.P. et al. Efeito da relação Ca:Mg do corretivo no desenvolvimento e nutrição mineral de mudas de aroeira (Myracrodruon urundeuva Fr. All.). Revista Cerne, v. 6, n. 1, p. 30-39, 2000. 